\title{
Food Security, Fertility Differentials and Land Degradation in Sub-Saharan Africa: A Dynamic Framework*
}

\author{
Maria Winkler-Dworak
}

\begin{abstract}
We study the impact of differential fertility levels for the food-insecure and food-secure population on the long-run values of the population distribution and resources in a descriptive model where the food security states are determined by a historically given food distribution and the endogenous food production with resources and labour as inputs. Furthermore, we assume that the resource stock is reduced by poverty-driven environmental degradation. Moreover, we incorporate nutritional effects on labour productivity and mortality. By applying local bifurcation theory, we show that the model may exhibit multiple equilibria. Furthermore, the orbits of resources and the population distribution may be characterised by quasi-periodic behaviour. Sustainable development in terms of approaching a steady state with positive values of resources and food-secure population is only promoted by low fertility levels of the food-insecure and food-secure population.
\end{abstract}

\section{Introduction}

"Over the past thirty years, most of sub-Saharan Africa has experienced very rapid population growth, sluggish agricultural growth, and severe environmental degradation" and there is evidence that these three phenomena are connected in a mutually reinforcing manner (Cleaver and Schreiber 1994). This nexus is commonly known as a 'vicious circle' in the literature.

The concept of the 'vicious circle of poverty' dates back to the 1950s, when Ragnar Nurkse observed that "in discussion of the problem of economic development a phrase that crops up frequently is the 'vicious circle of poverty'. [...] It implies a circular constellation of forces tending to act and react upon one another in such a way as to keep a poor country in a state of poverty (Ascher and Healy 1990). Similarly, Lutz and Scherbov (2000) describe the vicious circle model as being "based on the assumption that high fertility, poverty, low education and low status of

\footnotetext{
* This article is based on chapter 7 of my doctoral thesis "Population Growth, Food Security and Land Degradation: Modelling the Nexus in Sub-Saharan Africa."
} 
women and children are bound up in a web of interactions with environmental degradation and declining food production, in such a way that stress from one of these sources can trap certain rural societies, especially those living in marginal lands, into a vicious circle of increasingly destructive responses." Ascher and Healy (1990) tried to categorise this complex web of intervening factors into four distinct sectors: economic production, income distribution, natural resources and environment. ${ }^{1}$ They argue that in this rather complex web, various vicious circles involving two or more sectors may be observed (for examples see Ascher and Healy 1990, pages 20-23).

One example of such a vicious circle is illustrated by the parable of firewood (Nerlove and Meyer 1997). Gathering firewood, water, etc. is mainly the task of women and children. As deforestation proceeds, the borders of the forests are pushed further away from the village, so firewood collection becomes more time-consuming. Consequently, children become more valuable for their parents and the demand for them may rise, which reinforces fertility. Summing up, environmental degradation may itself increase fertility and subsequently population growth. ${ }^{2}$ Hence, Nerlove and Meyer (1997) argue that "[t]he relation between fertility and environmental degradation depends primarily on the way parents perceive the benefits of having children and not primarily on the effect of population size on the environment, as long as the environment is adversely affected by larger population". Similarly, Dasgupta (1993) finds that in poor countries children are also considered as income-earning assets which provides an additional motivation for having children.

In the spirit of the concept of the vicious circle, Lutz and Scherbov (2000) developed a quantitative simulation model, called PEDA (Population, Environment, Development and Agriculture), which links "population parameters and education to land degradation, food production and distribution" (Lutz and Scherbov 2000) and has been applied to several African countries, e. g., Burkina Faso, Cameroon, Madagaskar, Mali, Uganda and Zambia (Lutz et al. 2002).

The PEDA model is a population-based simulation model which considers, besides age and sex structure, the literacy and food-security status as well as the place of residence of the population. In sum, the PEDA model refers to about 1600 population states. It considers a natural resources module (land and water) as well as an agri-

1 However, Dworak (2002) argues that this categorisation is still incomplete since, e. g., polulation (including its age and sex distribution, fertility, mortalitiy, migration, education, etc.) and other institutional settings (security issues, property rights) are missing.

2 This controversial causal link has been tested empirically for data of Pakistan and Nepal by Filmer and Prittchett (1997) and Loughran and Pritchett (1997) in Asia. Filmer and Pritchett (1997) verify various effects enhancing the plausibility of the hypothesis that environmental scarcity could possibly raise the demand for children although there are several findings which may be inconsistent with it. However, Loughran and Pritchett (1997) reject the hypothesis, i. e., they find that environmental scarcity lowered the demand for children for Nepalese data. For sub-Saharan African cross-country data Cleaver and Schreiber (1994) find a positive relation between total fertility rates and the rate of deforestation. However, the significance is ambiguous (Cleaver and Schreiber 1994). 
cultural production module and it contains a food distribution mechanism in order to account for the inequality in the access to food.

In particular, the PEDA model includes the vicious circle reasoning. The growth of the rural (illiterate and food-insecure) population contributes to land degradation, lowering agricultural production and thereby increasing the number of food-insecure population. However, the PEDA model does not assume increasing fertility as a direct response to food insecurity because of its controversial empirical foundation. "Rather, the food-secure and food-insecure fractions of the population are assumed to have different fertility levels (subject to exogenously defined trends) and hence the aggregate fertility level only responds to changes in the food insecurity through changing weights of the groups in the calculation of overall fertility." (Lutz et al. 2004)

In this paper, we aim to investigate the assumption of differential fertility levels for the food-insecure and food-secure population in a reduced-form derivative of the PEDA model as presented in Lutz et al. (2002). ${ }^{3}$ Furthermore, we extend the PEDA framework by incorporating nutritional effects on labour productivity and mortality. In short, in each time period, the rural food-secure and food-insecure population together with the natural resource stock determine total food production. However, the population lowers the resource stock by land degradation. The total amount of food produced is distributed, in each time period, according to the prevailing food distribution function, thereby dividing the population into food-secure and food-insecure population.

The major part of the literature concerning differential fertility and income inequality is devoted to the context of investment in human capital. Although the focus in these studies lies on the long-run distribution of skilled to unskilled population, these papers are methodologically related to our reduced-form derivative of the PEDA model and may yield similar dynamic behaviour. For instance, Kremer and Chen (1999) show that the positive feedback between fertility differentials and income inequality may lead to multiple steady states of the long-run population distribution of skilled and unskilled population. In particular, they investigate the positive feedback between fertility differential and income inequality created by the fact that higher wages reduce fertility and that children of the unskilled are more likely to be unskilled than of the skilled.

Nevertheless, the model of Kremer and Chen differs substantially from our framework in several aspects. First, Kremer and Chen derive multiple steady states of the ratio of unskilled to skilled population, where the population growth rates are constant, but not necessarily equal to zero, in the steady states. Hence, Kremer and Chen (1999) consider a stable population rather than a stationary population in the

3 In Lutz et al. (2002) a comparison of the original PEDA model and its reduced-form derivative has been conducted. Surprisingly, the time paths of the food-insecure and food-secure population as well as the resources are quantitatively similar. Lutz et al (2002) conclude that "the reduced form model is adequate to investigate the role of the food distribution, and thereby contributes to a better understanding of the underlying mechanisms." 
equilibrium. Since we are considering a framework of limited resources and constant technology, the population has to become stationary in order to obtain a steady state.

Secondly, Kremer and Chen derive an inverse relationship between fertility rates and wages by assuming a simple utility function. Hence they define the population growth rates endogenously. In contrast, PEDA assumes different, but constant fertility levels for the food-insecure and food-secure population, respectively. Therefore, overall fertility rates vary only through compositional changes of the population.

Galor and Zeira (1993) explore the relationship between wealth distribution and investment in human capital, where the former evolves endogenously. They also find multiple equilibria in the long-run distribution of the skilled and unskilled population, although they assume zero population growth. However, the existence of the multiple steady states of the long-run distribution in their model basically depends on the assumption of technological non-convexity and imperfect credit markets (Galor and Zeira 1993). By comparing this assumption, involved in Kremer and Chen (1999), Galor and Zeira (1993) and others, to the PEDA assumptions and referring to earlier work, we aim to identify economic reasons for the resulting long-run distribution of food-insecure and food-secure population.

The remainder of the paper is organised as follows. Section 2 presents the model and discusses the underlying assumptions. Section 3 investigates the long-run evolution of resources, food-insecure and food-secure population. In particular, by applying bifurcation theory we analyse the effects of changing degrees of inequality and fertility differentials. Finally, section 4 concludes and presents further extensions.

\section{The model}

\section{Population dynamics}

At each time point $t$, population $P_{t}$ consists of two groups, the food-secure population, denoted by $P_{S t}$, and the food-insecure population, $P_{I t}$, with $P_{t}=P_{S t}+P_{I t}$. Let us denote the threshold level $\tilde{y}$ as the minimum level of calories necessary to be food secure. Then, a person belongs to group $P_{S t}$ or $P_{I t}$, respectively, if he or she receives a level of food that exceeds, or falls short of, $\tilde{y}$. Let $G\left(\tilde{y} ; y_{t}\right)$ denote the share of the population that falls short of the subsistence requirement based on a per capita food entitlement $y_{t}$ in the period $t$. Then, the food-insecure population at time $t+1$ is given by $G\left(\tilde{y} ; y_{t}\right)$, times the existing population, $P_{t}$, plus births and deaths of individuals in the food-insecure population at time $t$, i. e.,

$$
P_{I, t+1}=\left(b_{I}-d\left(y_{I t}\right)\right) P_{I t}+G\left(\tilde{y} ; y_{t}\right) P_{t}
$$

where $b_{I}$ and $d\left(y_{I t}\right)$ denote the birth rate and the death rate for the food-insecure population, respectively. A similar equation can be derived for the food-secure population, i. e., 


$$
P_{S, t+1}=\left(b_{S}-d\left(y_{S t}\right)\right) P_{S t}+\left(1-G\left(\tilde{y} ; y_{t}\right)\right) P_{t},
$$

where $b_{S}$ and $d\left(y_{S t}\right)$ denote the birth rate and the death rate for the food-secure population, respectively. Contrary to the birth rates, which are exogenously given, we assume that the death rates of the food-insecure and food-secure population depend on the per capita food entitlement of the respective sub-population, $y_{I t}$ and $y_{S t}$, with mortality decreasing when food entitlement rises, i. e., $d^{\prime}<0$. $^{4}$

In particular, we assume the following functional form for the crude death rates

$$
d\left(y_{j}\right)=d_{n a t}+\frac{d_{\max }}{1+\exp \left(\delta\left(y_{j}-y_{m}\right)\right)}, \quad \text { for } j=I, S,
$$

which has been proposed in Strulik (1995) and has also been used in Prskawetz et al. (2000). In formula (3), $d_{\text {nat }}$ indicates a minimum death rate which is approached for values of the per capita food entitlement rising to infinity. Furthermore, $d_{n a t}+d_{\max }$ are approximately the maximum death rate for a per capita food entitlement close to zero. Within these upper and lower bounds of mortality, the crude death rates fall with increasing per capita food entitlement, where the rate of decrease is initially rising and then decreasing. Furthermore $\delta$ measures the slope of the mortality function. Finally, if the per capita food entitlement equals $y_{m}$, mortality amounts to about half of its maximum value (Strulik 1995).

Moreover, it seems reasonable to assume that $b_{I}, b_{S}<d_{\text {nat }}+d_{\text {max }}$ holds, which implies that for a per capita food entitlement close to zero, the natural growth rates of the sub-populations are negative.

\section{Food distribution}

At the end of each time period the total amount of food available $Y_{t}$ is distributed among the population. We postulate a historically given food distribution function which is represented by a Lorenz curve in order to be consistent with the original PEDA model. ${ }^{5}$ This curve plots cumulative shares of food $L(F(z))$ as a function of cumulative population shares $F(z)$ when the individuals are ranked in increasing order of the food $z$ that they receive. For instance, $L\left(P_{I t} / P_{t}\right)$ indicates the share of total food

4 In what follows, the prime after function names denotes the first derivative and the double prime the second derivative.

5 Atkinson (1970), Rothschild and Stiglitz (1973) and Anand (1983, citet in Sen (1997)) showed that a broad range of measures of inequality are Lorenz consistent, which means that they agree with the Lorenz quasi-ordering when it applies (i. e., Lorenz curves for alternative income distributions do not intersect). Therefore by using the concept of Lorenz dominance, we ensure that this measures agree about the ordering of the food distribution and our results are not specific to the measure of inequality used. 
which the food-insecure population receives. ${ }^{6}$ The food distribution function together with the total amount of food $Y_{t}$ to be distributed in each time period and the threshold level of food $\tilde{y}$ then determine the share of food-secure $P_{S, t+1}$ and food-insecure people $P_{I, t+1}$, respectively, in the following period.

Recalling some basic mathematical properties of the Lorenz curve, we can derive an analytical expression for the population share falling short of the minimum requirement in each time period. We exploit the fact that the slope of the Lorenz curve at any point $F(z)$ is inversely proportional to the per capita food production $y=Y / P$ and proportional to the corresponding food level $z$ (see Appendix A.1 for the derivation of this result)

$$
\frac{d L(F(z))}{d F(z)}=l(F(z))=\frac{1}{y} z
$$

Assuming further that we can analytically solve for the inverse of the derivative of the Lorenz curve, equation (4) can be written as

$$
F(z)=l^{(-1)}\left(\frac{z}{y}\right)
$$

Since $F$ represents a distribution function, it has to be constrained by one from above. Obviously, the corresponding level of food $z_{\max }$ for which $F\left(z_{\max }\right)=1$ holds first, indicates the maximum level of food entitlement in the economy. Eq. (5) then implies that the maximum level of food will depend on the prevailing level of per capita food production $y$ and the functional form of the Lorenz curve. Unless the economy is in a stationary state, per capita food production $y$ and henceforth the maximum level of food $z_{\max }$ will vary over time.

For the numerical analysis we postulate the following simple functional form for the Lorenz curve ${ }^{7}$ :

$$
L(F(z))=(F(z))^{\alpha} \quad \alpha>1 .
$$

This simple Lorenz curve fulfills the assumption that the first derivative is analytically invertible, which has been postulated above. Moreover, it has the advantage that a single parameter, namely $\alpha$, uniquely governs the degree of inequality and therefore ranks the food distributions.

However, the specific functional form (6) for the Lorenz curve implicitly assumes a zero minimum food entitlement. Since the minimum requirement of calories in order to be food secure is strictly positive, there will always be a strictly positive num-

6 Hence, the per capita food entitlement at time $t$ of the of the food-insecure population is defined as $y_{I t}=Y_{t} L\left(P_{I t} / P_{t}\right) / P_{I t}$. Furthermore, the per capita food entitlement at time $t$ of the food-secure population is given by $y_{S t}=Y_{t}\left(1-L\left(1-P_{S t} / P_{t}\right)\right) / P_{S t}$.

7 See Chotikapanich (1993) for alternative functional forms of the Lorenz curve. 
ber of people falling short of this minimum requirement. Hence, we will not obtain a steady state with a zero number of food-insecure population.

By using this specific form of the Lorenz curve, equation (5) transforms to

$$
F(z)=\left(\frac{z}{\alpha y}\right)^{1 /(\alpha-1)} .
$$

Recalling that $\tilde{y}$ indicates the threshold level of food, a person needs to be food secure, equation (7) evaluated at $z=\tilde{y}$ gives the proportion of the population becoming food insecure, i. e.

$$
G\left(\tilde{y} ; y_{t}\right)= \begin{cases}\left(\frac{\tilde{y}}{\alpha y_{t}}\right)^{\frac{1}{\alpha-1}} & \text { for } \alpha y_{t}>\tilde{y} \\ 1 & \text { otherwise. }\end{cases}
$$

Note that when $\tilde{y}$ exceeds the maximum food entitlement $z_{\max }=\alpha y_{t}$, then $G\left(\tilde{y} ; y_{t}\right)$ equals one and the entire population will become food insecure in the next period.

Food is produced according to a Cobb-Douglas production function with the inputs agricultural labour, represented by the rural population, and resources. ${ }^{8}$ Aggregation over the exogenous production factors and production adjustments of the PEDA model, as outlined above, yields the production function

$$
Y_{t}=T\left(h_{I} P_{I t}+h_{S} P_{S t}\right)^{\beta_{1}} R_{t}^{\beta_{2}}, \quad h_{I}+h_{S}=1,
$$

where $h_{I}$ and $h_{S}$ denote constant values of labour efficiency of the food-insecure and food-secure population, respectively. Furthermore, we normalise the efficiency units and we assume that $h_{I}<h_{S}$. The latter assumption is based on the fact that "a person's consumption-intake affects his productivity" (Dasgupta and Ray 1987). ${ }^{9}$ Furthermore, $T$ indicates the constant technology parameter and $R_{t}$ represents the resource stock at time $t$. In addition, $\beta_{1}$ and $\beta_{2}$ denote the production elasticities with respect to labour and resources, respectively.

8 In this analysis, we restrict ourselves to food crops, abstaining from cash crops. Hence, markets are not modelled.

9 An empirical confirmation of the nutrition-productivity hypothesis using household-level data from Sierra Leone is provided in Strauss (1986). 


\section{Resource dynamics}

The stock of resource increases by indigenous growth/regeneration and is lowered by poverty-driven land degradation, i. e.

$$
R_{t+1}=R_{t}+g\left(R_{t}\right)-D\left(P_{I t}, P_{t}, R_{t}\right)
$$

where we assume a declining rate of regeneration. This assumption is based on a sketch of the exploitation during cropping and the natural regeneration during the fallow period of tropical soil in Ruthenberg (1980, p. 62). In this sketch, the fertility of soil increases concavely over time during fallow. ${ }^{10}$ That means, the higher the resource stock, the lower the rate of regeneration. Hence, we assume a saturation level or maximum level of the resource stock, $\bar{R}$, which is the stationary solution of $R$ if the resource is not degraded. In particular, we assume that regeneration can be represented by the difference of the maximum level, $\bar{R}$, and the actual resource stock, $R_{t}$, times the speed at which the resource regenerates, $a$ :

$$
g\left(R_{t}\right)=a\left(\bar{R}-R_{t}\right)
$$

In addition, we assume that resource degradation hinges on the magnitude of food-insecure population and on the stock of available resources. More specifically, we postulated that the degradation function is increasing in all arguments. In particular Lutz et al. (2002) assume

$$
D\left(P_{I t} P_{t}, R_{t}\right)=\gamma \frac{P_{t}}{\bar{R}} P_{I t} \frac{R_{t}}{R_{t}+\eta},
$$

where $\gamma$ and $\eta$ are fixed parameters. While it is assumed that degradation increases linearly in $P_{I}$, degradation is modelled to rise at a decreasing rate with the level of the resources. This assumption reflects the fact that the higher the available resource stock, the lesser the environmental stress. Furthermore, degradation is zero if the resources are completely degraded. Similarly, if the entire population is food secure, the resources will not be degraded. "Land degradation is by no means caused only by the poor. Irresponsible rich farmers sometimes exploit the land, but by and large, farmers with secure tenure and capital are more likely to conserve natural resources. When natural disasters occur they can turn to alternative sources of income, borrow and repay in better years. These alternatives are not open to the poor" (Young 1998).

10 The logistic growth function, which is commonly used in such a context (e. g., see Clark 1990, Brander and Taylor 1998, Anderies 1998, 2003) would rather imply a S-shaped curve over time. 
The focus of this paper is to analyse how the vicious circle of poverty and environmental degradation can be broken. Therefore, we only model poverty-driven environmental degradation and disregard differently motivated degradation. ${ }^{11}$

Moreover, degradation is scaled by population density in order to reflect the fact that poverty-induced environmental degradation is reinforced by population pressure. Dworak (2002) conducts a sensitivity analysis of the results with respect to the functional form of the degradation function in a framework of zero population growth. Surprisingly, the results show to be rather robust with respect to the functional form of the resource equation. Furthermore, Dworak (2002, chapter 8) reformulates the resource equation in order to incorporate soil quality and quantity explicitly, as they are affected differently by population pressure.

\section{Calibration}

The parameter values used in the remainder of this paper are set according to Lutz and Scherbov (2000), who calibrated their model to data of Mali, except that the degree of inequality in the food distribution, $\alpha$, and the technology parameter $T$ were chosen such as to highlight the complexity of the dynamics. Therefore, the situation of Mali will be separately discussed in the conclusions. However, the model studied here extends the PEDA framework by incorporating nutritional mortality effects. The calibration of the parameter values of the mortality function and the assumptions about fertility are described below.

The time series data necessary to estimate the parameters $d_{n a t}, d_{\max }$ and $\delta$ are sparse or difficult to obtain, if they even exist. For instance, the reports about the state of food insecurity (Food and Agriculture Organization 1999, 2000, 2001), which have appeared annually since 1999, published the depth of food insecurity for the single countries for 1996-98 only in 2000. Hence, there are only estimates for the per capita dietary energy supply of the food-insecure and food-secure population for 1996-98. Moreover, there are no data at all about mortality by food security status. However, the latter constraint can be overcome by writing the crude death rate of the total population as weighted average of the crude death rates of the sub-populations, where the weights are given by the share of the respective sub-population, which are monitored annually by the Food and Agriculture Organisation of the United Nations, i. e.

$$
\begin{gathered}
d_{t}=d\left(y_{I t}\right) \frac{P_{I t}}{P_{t}}+d\left(y_{S t}\right)\left(1-\frac{P_{I t}}{P_{t}}\right) \\
=d_{n a t}+d_{\max }\left(\frac{P_{I t} / P_{t}}{1+\exp \left(\delta\left(y_{I t}-y_{m}\right)\right)}+\frac{1-P_{I t} / P_{t}}{1+\exp \left(\delta\left(y_{S t}-y_{m}\right)\right)}\right) .
\end{gathered}
$$

11 For the modelling of resource degradation driven by other motives (cultural, profit maximisation, etc.) see e. g., Clark (1990), Brander and Taylor (1998), and Anderies (1998, 2003), where the natural resources range from fish to trees to soil. 
Figure 1. Graphical representation of the postulated mortality function

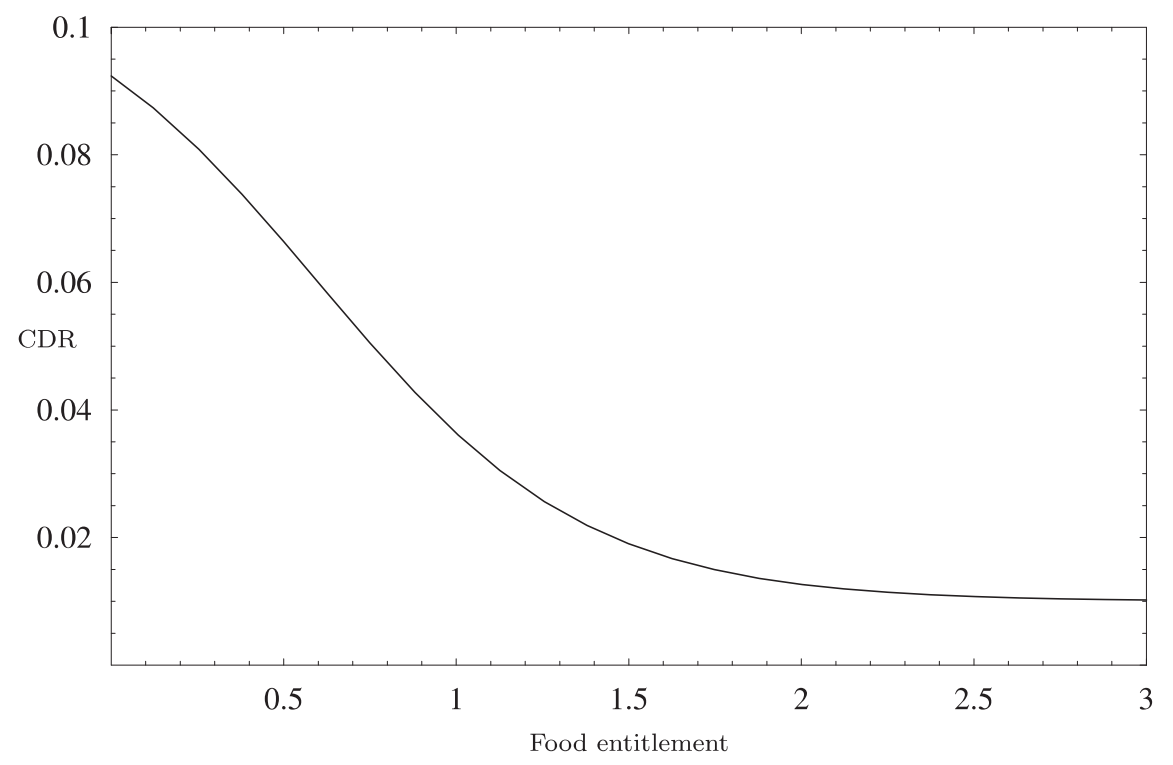

Nevertheless, the data sets are too sparse in order to obtain reliable parameter estimates. Therefore, we have to base our parameter estimations primarily on assumptions than on real data. For instance, Strulik (1995) assumes that $d_{n a t}$ and $d_{\max }$ equal 0.01 and 0.1 , respectively, which seem plausible for our purpose as well. Furthermore, it seems reasonable to assume that $y_{m}$ is less than the minimum requirement of calories in order to be food secure. For the following simulations we postulate that $y_{m}$ equals 0.6 , which is half the minimum requirement in order to be food secure. Combining these assumptions with the estimates for the crude death rate of the total population, the share of the food-insecure population, and the per capita dietary energy supply for the food-insecure and food-secure population, respectively, yields an estimate for the slope of the mortality function, $\delta$, of about 2.57 . Fig. 1 plots the mortality function under the assumption of these parameter values.

Moreover, no time series data of fertility by food security status are available. Hence, the fertility differential between the food-insecure and food-secure populations has to be based on assumptions.

Finally, Table 1 summarises the parameter values used. As noted above, data are sparse and if they are available, their reliability has to be often questioned. ${ }^{12}$ Thus the numerical simulations require a broad sensitivity analysis, which will be given in the next section.

12 For a discussion on data availability and quality for Sub-Saharan Africa see Dworak (2002). 
Table 1. Summary of parameter values.

\begin{tabular}{|c|c|c|}
\hline Parameter & Value & Description \\
\hline$a$ & 0.02 & Indigenous growth rate of resources \\
\hline$\alpha$ & 1.3 & Degree of inequality \\
\hline$b_{I}$ & 0.05 & Crude birth rate of the food-insecure population \\
\hline$b_{S}$ & 0.03 & Crude birth rate of the food-secure population \\
\hline$\beta_{1}$ & 0.534 & Production elasticity with respect to labour \\
\hline$\beta_{2}$ & 0.088 & Production elasticity with respect to resources \\
\hline$d_{\text {nat }}$ & 0.01 & Crude death rate independent from food entitlement \\
\hline$d_{\max }$ & 0.1 & Maximum crude death rate which depends on food entitlement \\
\hline$\delta$ & 2.57 & Slope of the mortality function \\
\hline$\eta$ & 0.67 & Concavity parameter of the degradation function \\
\hline$\gamma$ & 0.0089 & Degradation impact factor \\
\hline$h_{I}$ & 0.2 & Labour efficiency of the food-insecure population \\
\hline$h_{S}$ & 0.8 & Labour efficiency of the food-secure population \\
\hline $\bar{R}$ & 1 & Maximum resource stock \\
\hline$T$ & 2.28 & Technology parameter of the production function \\
\hline$\tilde{y}$ & 1.2 & Minimum requirement of calories \\
\hline$y_{m}$ & 0.6 & Food entitlement, where the crude death rate reaches half of its maximum value \\
\hline
\end{tabular}

\section{Dynamics and bifurcation analysis}

Putting the pieces together, the dynamical system studied in this paper is given by

$$
\begin{gathered}
P_{I, t+1}=\left(b_{I}-d\left(y_{I t}\right)\right) P_{I t}+G\left(\tilde{y} ; y_{t}\right)\left(P_{I t}+P_{S t}\right) \\
P_{S, t+1}=\left(b_{S}-d\left(y_{S t}\right)\right) P_{S t}+\left(1-G\left(\tilde{y} ; y_{t}\right)\right)\left(P_{I t}+P_{S t}\right) \\
R_{t+1}=R_{t}+a\left(\bar{R}-R_{t}\right)-\gamma \frac{P}{\bar{R}} P_{I t} \frac{R_{t}}{R_{t}+\eta} .
\end{gathered}
$$

In the remainder of the paper, we will proceed as follows. First, we compute the steady states of the difference equation system (13)-(15). By applying local bifurcation theory, we determine regions of different long-run evolution of the orbits (i. e., multiple equilibria, quasi-periodic orbits) in the parameter space in order to identify the long-run effects of parameter changes.

Proposition 1 If the natural population growth rate of the food-insecure population for a per capita food entitlement of $\tilde{y}$ / $\alpha$ is positive, then there exists a non-trivial steady state where the entire population is food insecure. 
The proof is given in Appendix A.2. Proposition 1 can be intuitively explained as follows. A non-trivial steady state, where the entire population is food insecure requires that $G\left(\tilde{y} ; y_{t}\right)$ equals one and the natural population growth rate of the food-insecure population, $b_{I}-d\left(y_{I t}\right)$, equals zero for all $t$. If the entire population is food insecure, then even the maximum food entitlement, which equals $\alpha y_{t}$ assuming the functional form given in Eq. (6) for the Lorenz curve, is less than the minimum requirement $\tilde{y}$, or equivalently,

$$
y_{I t}=y_{t}<\frac{\tilde{y}}{\alpha} \quad \forall t
$$

Note that if the entire population is food insecure, the per capita food entitlement of the total population is per definition equal to the per capita food entitlement of the food-insecure population. It follows from Eq. (16) that the natural growth rate of the population which is entirely food insecure has to be less than the hypothetical natural population growth rate for a per capita food entitlement of $\tilde{y} / \alpha$, i. e.,

$$
b_{I}-d\left(y_{t}\right)<b_{I}-d\left(\frac{\tilde{y}}{\alpha}\right) .
$$

If the right-hand side of Eq. (17) is negative, the natural population growth rate would always be negative, which contradicts the requirements of a non-trivial steady state. Hence

$$
b_{I}-d\left(\frac{\tilde{y}}{\alpha}\right) \geq 0
$$

is a necessary condition for the existence of a non-trivial steady state, where the entire population is food-insecure. Furthermore, if equality holds in condition (18), it determines the critical parameter values, where this steady state ceases to exist and the long-run share of food-insecure population may fall below one.

However, whether there exist further fixed points with an equilibrium share of the food-insecure population less than one can only be determined numerically.

In Fig. 2 and 5, we investigate numerically the long-run behaviour of the dynamical system (13)-(15) by jointly changing the degree of inequality and the fertility differential, as well as the crude birth rates, respectively. In particular, there exist either one or three fixed points. As evident from Fig. 2 and 5, the orbits may become quasi-periodic in the long run as represented by a closed invariant curve in the state space. $^{13}$

In particular, in Fig. 2 the effect of a jointly changing degree of inequality and the fertility differential between the food-insecure and food-secure population is investigated, where we fix the crude birth rate of the food-insecure population. At $\alpha=1.584$, which corresponds to

13 For technical details on the bifurcation analysis see Appendix A.3. 


$$
b_{I}-d\left(\frac{\tilde{y}}{\alpha}\right)=0
$$

for the parameter values given in Table 1, the long-run behaviour changes dramatically. In particular, there exists only one fixed point, which is stable (region (d) ) or unstable and surrounded by a stable closed invariant curve (region (e)). In contrast for $\alpha$ less than this threshold, it is also possible that three fixed points exist (regions (b) and (C)), where the equilibrium number of food-secure population equals zero for one of the fixed points. Furthermore, the latter fixed point is always stable, where in region (b) there exists a second stable fixed point, which is unstable in region (c). This second equilibrium exhibits a high equilibrium resource stock and a relatively low population size, where the equilibrium share of the food-insecure population is low.

As noted above in region (e), the unique fixed point is unstable and the orbits converge to a closed invariant curve giving rise to quasi-periodic long-run behaviour. Fig. 3 visualises the latter in the population-resources state space, where the corresponding share of the food-insecure population is indicated by the brightness of the curve. Furthermore, Fig. 4 shows the time series on the closed invariant curve. The dynamics can be summarised as follows: if the whole population is food insecure, the total population will decline and resources can regenerate. Once a sustainable level of resources and population has been restored, the food-insecure population declines. But while this share of the population declines, the total population will grow in turn, which will again jeopardise the resources. As resources decrease, the numbers of food-insecure people increase again until the entire population is food insecure and the 'cycle' starts over again. However, since the orbits on the closed invariant curve are only almost periodic, the shape of the time series is not entirely conform over time. 
Figure 2. Bifurcation diagram with respect to the degree of inequality, $\alpha$ and the fertility differential between the food-insecure and food-secure population. All other parameters are set as in Table 1. The dot indicates the parameter values of $\alpha$ and the fertility differential, $b_{I}-b_{s}$, assumed in Table 1. The dashed curve represents the pair of parameter values of $\alpha$ and the fertility differential, $b_{I}-b_{s}$, where track of the stable closed invariant curve is lost. For technical details on bifurcation analysis see Appendix A.3.

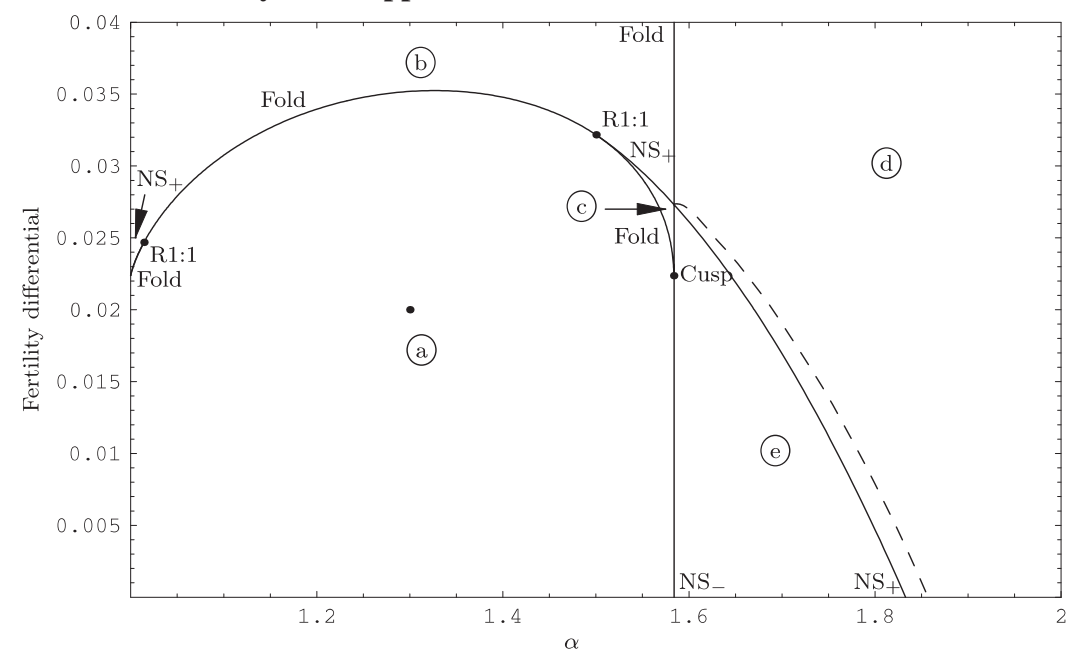

(a) Unique fixed point with $\hat{P}_{S}=0$

(b) Three fixed points: two stable, where one exhibits $\hat{P}_{S}=0$, and a saddle.

(c) Three fixed points: one stable, which exhibits $\hat{P}_{S}=0$, and two saddles.

(d) Unique stable fixed point, with $\hat{P}_{S}>0$.

(e) Unique unstable fixed point surrounded by a stable closed invariant curve.

Figure 3. Graphical representation of the closed invariant curve in the state space of the population and resources. The share of the food-insecure population is indicated by the brightness of the curve.

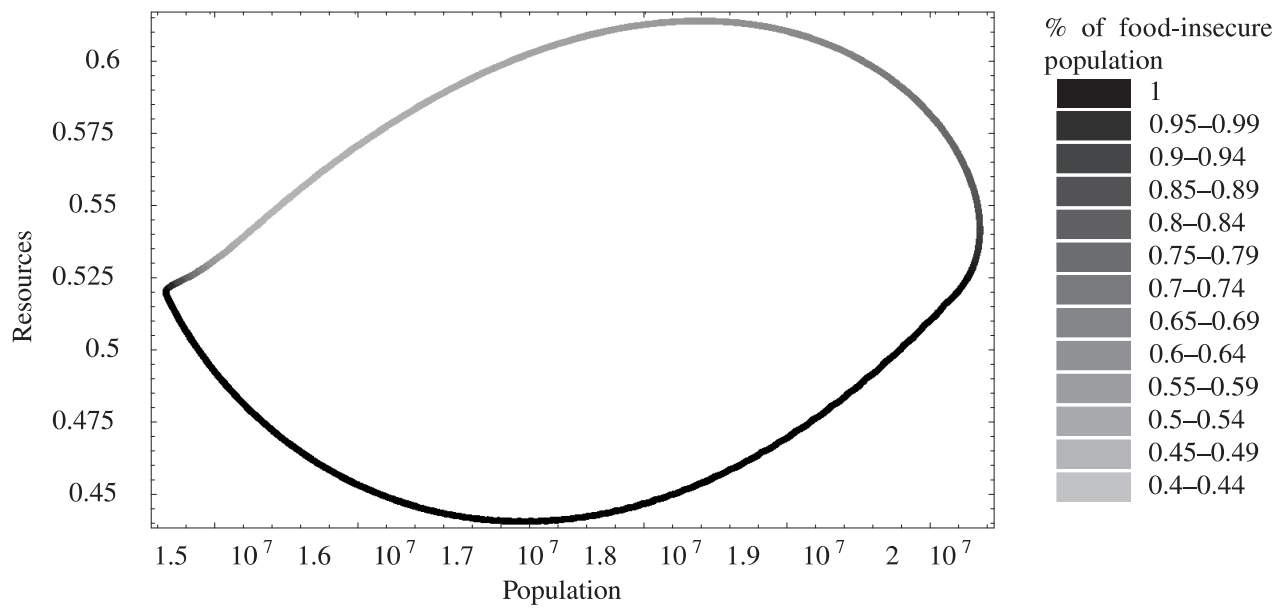


Moving from the left to the right in Fig. 2 the distribution effect, which is extensively discussed in Prskawetz et al. (2003), can be detected. In particular, Prskawetz et al. (2003) investigate the effect of the inequality in the distribution of food in the PEDA framework under the assumption of zero population growth and constant resources. Thereby, they split the ambiguous effect of varying the degree of inequality in a distribution and a production effect. In particular, an increase in the degree of inequality, ceteris paribus, will intuitively increase the share of the food-insecure population as more food is distributed to the upper classes. However, Prskawetz et al. (2003) demonstrate that this must not necessarily be true. It may happen that the share of food-insecure population even decreases, as the degree of inequality increases. ${ }^{14}$ In this case, food entitlement of the poorest of the poor is shifted to the upper food entitlement classes, thereby increasing also the food entitlement of those who just fall short of the minimum requirement.

Figure 4. Graphical representation of the orbits on the closed invariant curve.
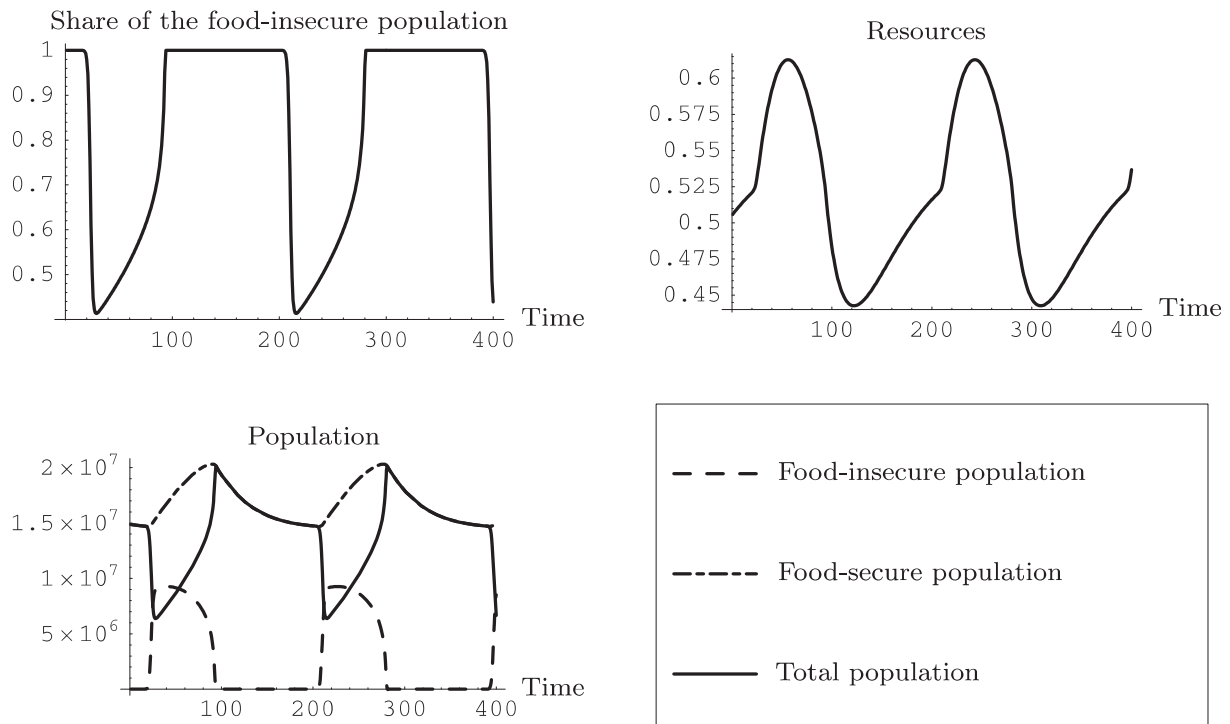

In addition, a change in the degree of inequality also effects the maximum food entitlement $\alpha y$ and henceforth determines whether the entire population will be food insecure in the long run. In particular, as more food is distributed to the upper food entitlement classes, the maximum food entitlement of the economy will rise (distribution effect). However, as the number of food-insecure population varies due to a change of the degree of inequality, the total amount of food produced and henceforth

14 Prskawetz et al. (2003) also demonstrate that the existence of such a result can be shown for a general food distribution function by using mean preserving spreads as introduced by Rothschild and Stiglitz (1970). 
the maximum food entitlement vary, too (production effect). If the share of the less efficient food-insecure people increases as a consequence of a rising degree of inequality, the total amount of food produced declines and thus lowering the maximum food entitlement. Summing up, if a negative production effect dominates the distribution effect, the maximum food entitlement decreases and the probability that the entire population will be food insecure in the long run, increases. However, if the distribution effect dominates, then at least the richest person is entitled to a sufficient amount of food to be food secure in the long run. Hence, the equilibrium share of the food-insecure population falls below one if the degree of inequality is sufficiently increased (cf. Fig. 2 as $\alpha$ passes from region (a) to (e)).

Moving in the left part of Fig. 2 from the bottom to the top (i. e., from region (a) to (b) a second stable equilibrium emerges. In this case, the fertility level of the food-secure population is sufficiently lower than that of the food-insecure population implying that the natural growth rate of the food-secure population may turn negative and there may be a positive number of food-secure people in the long run. However, the negative natural population growth rate for the food-secure population implies a continuous flow from the food-insecure to the food-secure population in the equilibrium. Hence, for the existence of such an equilibrium a high resource stock together with a low total population size is required.

However, the results presented in Fig. 2 crucially depend not only on the fertility differential between the food-insecure and the food-secure population but also on the fertility level of the food-insecure population, which was held fixed so far. Hence, Fig. 5 illustrates the change of the long-run behaviour as the crude birth rates of the food-insecure and food-secure population are varied simultaneously. For high fertility of the food-insecure population, everybody will be food insecure in the long run. If the crude birth rate of the food-secure population is sufficiently low, a second fixed point with a low total population size but with a positive share of the food-secure population emerges. A low crude birth rate of the food-secure population in the presence of high fertility levels of the food-insecure population may decrease the share of the food-insecure population in the long run but simultaneously implies also a low total population in equilibrium. The long-run behaviour drastically changes if the crude birth rate of the food-insecure population falls below a hypothetical death rate for a per capita food entitlement of $\tilde{y} / \alpha$ for the food-insecure population, i. e.,

$$
b_{I}<d\left(\frac{\tilde{y}}{\alpha}\right)
$$

which corresponds to $b_{I}<0.04$ in Fig. 5. Then there is either a stable fixed point with a positive number of food-secure population or, for higher fertility levels of the food-secure population, there is an unstable fixed point surrounded by a stable closed invariant curve as illustrated in Fig. 3 and 4. Consequently, low fertility levels of both sub-populations promote sustainable development. However, we did not consider crude birth rates below 0.01 in our analysis, since for such low fertility rates the population will be extinct in the long run. Proposition 2 summarises the findings. 
Proposition 2 Low fertility levels of the food-secure population in the presence of high crude birth rates of the food-insecure population increase the chances for sustainable development by eventually decreasing the long-run share of the food-insecure population but simultaneously imply a low total population in equilibrium. Only low fertility levels of both sub-populations yield sustainable development for all initial values.

Figure 5. Bifurcation diagram with respect to the crude birth rates of the food-insecure and food-secure population. All other parameters are set as in Table 1. The dot indicates the parameter values of $b_{S}$ and $b_{I}$ assumed in Table 1 . The dashed curve represents the pair of parameter values of the birth rates $b_{s}$ and $b_{l}$, where track of the stable closed invariant curve is lost. For technical details on bifurcation analysis see Appendix A.3.

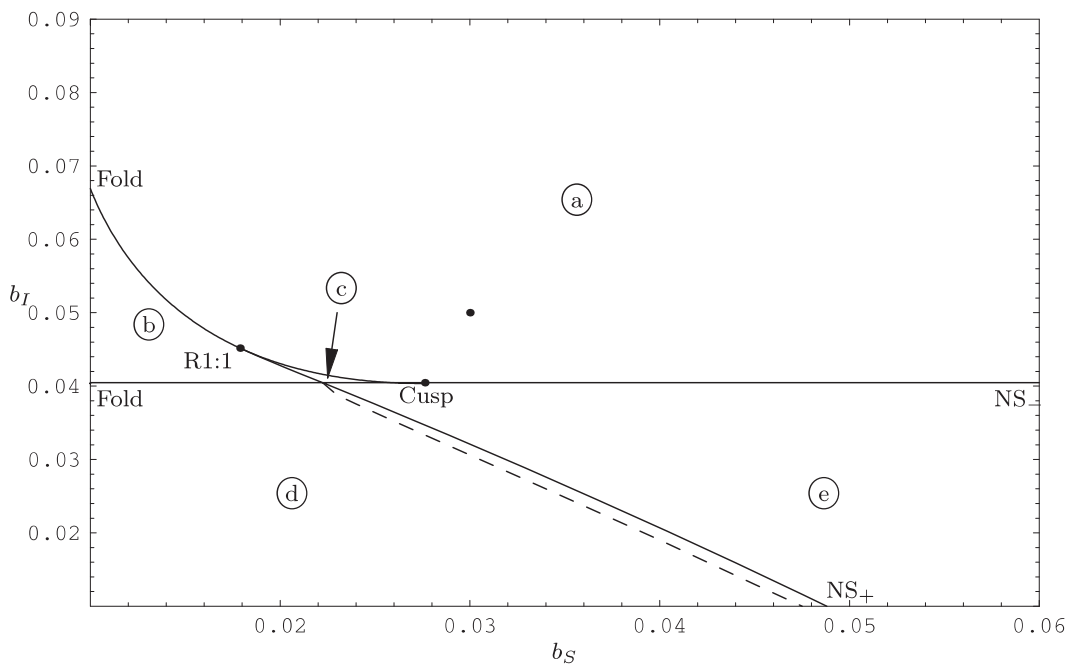

(a) Unique fixed point with $\hat{P}_{S}=0$

(b) Three fixed points: two stable, where one exhibits $\hat{P}_{S}=0$, and a saddle.

(c) Three fixed points: one stable, which exhibits $\hat{P}_{S}=0$, and two saddles.

(d) Unique stable fixed point, with $\hat{P}_{S}>0$.

(e) Unique unstable fixed point surrounded by a stable closed invariant curve.

\section{Conclusions and extensions}

This paper introduces and analyses a dynamic model of population and natural resources, where the population is split into two sub-groups according to their food security status. The latter is determined by a historically given food distribution and by the endogenous food production with agricultural labour and natural resources as inputs, where the resource stock is reduced by poverty-driven land degradation. Moreover, we incorporate nutritional effects on labour productivity and mortality and we assume different fertility levels for the food-insecure and food-secure population.

As noted earlier, this model is calibrated according to data from Mali, except for 
the degree of inequality and the technology parameter. In particular, Mali exhibits a degree of inequality in the distribution of food that is about twice as high as our baseline level given in Table 1. Consulting Fig. 2, Mali would lie rather in region (d), where there exists a unique stable fixed point with an equilibrium share of the food-insecure population less than one. Furthermore, the baseline level of the technology parameter is somewhat lower than the estimate used in the original PEDA model (Lutz and Scherbov 2000). A higher value of the technology parameter, ceteris paribus, would yield higher food production levels and a lower share of food-insecure population. Hence, our model does not predict Mali to be doomed for the future.

But as noted above, data to estimate parameters are sparse, and even if they are available, their reliability may be questioned. In particular, there are no time series data, to our knowledge, on fertility by food security status. Therefore the estimate of the parameter values of the fertility differential between food-insecure and food-secure population has so far been based rather on assumptions. If our assumptions were too optimistic, our model may predict that Mali would be locked in long-run oscillations where the entire population is repeatedly food insecure.

From a methodological perspective, our framework is closely related to the work dealing with income distribution and differential fertility in the context of investment in human capital.

For instance, Kremer and Chen (1999) show that the positive feedback between fertility differentials and income inequality may lead to multiple steady states of the long-run distribution of skilled and unskilled population. Translating the skilled and unskilled population into food-insecure and food-secure population, where labour productivity depends on the nutritional status, enables us to compare our results to Kremer and Chen (1999), Galor and Zeira (1993) and others.

As noted earlier, the model of Kremer and Chen (1999) differs substantially from our framework by the assumption of endogenous fertility and that they are considering steady states of the ratio of unskilled to skilled population rather than steady states of the stocks of the unskilled and skilled population. Recalling that we are considering a framework of limited resources and constant technology, the population has to become stationary in order to obtain a steady state.

In contrast to the assumption of endogenous fertility by Kremer and Chen (1999), PEDA considers different, though constant, fertility levels for the food-insecure and food-secure population, respectively. Therefore, overall fertility rates vary only through compositional changes of the population.

However, what Kremer and Chen (1999) and the PEDA framework have in common is that they impose an exogenously given distribution of education costs in the case of Kremer and Chen (1999) and of food in the PEDA model. This exogenously defined distribution mainly governs the dynamics of the long-run population distribution.

In contrast, Galor and Zeira (1993) explore the relationship between wealth distribution and investment in human capital in an overlapping generations framework 
with intergenerational altruism, where the wealth distribution endogenously evolves over time via bequests. They also find that the long-run distribution of skilled and unskilled workers depend on the initial wealth distribution. However, Galor and Zeira (1993) derive multiple equilibria of the long-run distribution of the skilled and unskilled population in a framework of zero population growth. Similarly, Dworak (2002, chapter 5) finds multiple steady states of the long-run distribution of the food-insecure and food-secure population in the reduced-form derivative of the PEDA model under the assumption of zero population growth for both sub-populations. Hence in Galor and Zeira (1993) and Dworak (2002, chapter 5), multiple equilibria are mainly generated by the positive feedback between the income/food distribution and labour productivity depending on the educational/nutritional status within a framework of stationary populations.

Maintaining the assumption of differential population growth for the food-insecure and food-secure population but setting aggregate population growth to zero, Dworak (2002, chapter 6) identifies that differential population growth only affects the domain of attraction of the respective steady states. Adding aggregate, exogenous population growth reduces the number of equilibria to a unique steady state. However, this unique steady state may be unstable and the orbits either tend to unlimited population growth while resources become extinct or total population size approaches zero while resources tend to their maximum level in the long run (Dworak 2002, chapter 6). Assuming endogenous death rates prevents that the population grows unlimited in the long run and henceforth allows multiple equilibria again. Moreover, if the exogenous birth rate exceeds the exogenous death rate of the food-insecure population, the orbits may become quasi-periodic in the long run (Dworak 2002, chapter 6). The latter results also hold for endogenous mortality. Hence, adding aggregate population growth may cause oscillations of the population stock, the numbers of food-insecure population and the resources in the long run.

The aim of this paper was to identify the role of differential fertility of the food-insecure and food-secure population within a framework of unequal access to food. We find out that a higher fertility differential in terms of the difference of the fertility level of the food-insecure population minus that of the food-secure population may decrease the steady state numbers of food-insecure population, but the latter is mainly driven by the degree of inequality and the respective fertility levels. In particular, only low fertility levels for both sub-populations promote sustainable development in terms of positive levels of resources and population and independent of the initial distribution of food-secure versus food-insecure population.

These results highlight the role of population growth as it is linked to poverty. As we demonstrate, food insecurity may even persist in a stationary population with an unequal distribution of food, where population growth exacerbates poverty. In our framework, fertility reductions of the food-insecure and food-secure population mitigate the stress on resources and subsequently on food production. The latter result is a prominent claim of classical economics. However, it is even acknowledged by some of the neoclassical economists, who regard high population growth as a neutral 
factor, "that fertility reduction can buy time while resource substitutes are found or market or institutional inefficiencies are addressed" (Jolly 1994).

In the PEDA framework, the institutional settings are only reflected by the assumption of a historically given unequal food distribution. However, no justification is given for the underlying assumptions about the institutions (e. g., property regimes, access to markets), although the institutional settings reflect the transition between the food-security states as well as the resource equation, particularly, the degradation function. Beaumont and Walker (1996) nicely address the latter, investigating the optimal choice of labour input and intensity of farming under different property regimes. Dworak (2002, chapter 8) proposes a reformulation of the resource equation by distinguishing between quantity and quality of soil as they are reflected differently by population pressure. A combination of these two approaches would substantially improve the modelling of the resource side in the PEDA framework.

Another drawback of the PEDA framework is that the degree of inequality is held constant over time. Galor and Zeira (1993) present an endogenously evolving wealth distribution by the means of micro-foundation of bequests. The incorporation of bequests together with the property regimes would make the PEDA framework much more realistic.

\section{Acknowledgements}

I am grateful to Alexia Prskawetz, Warren Sanderson, my supervisor Gustav Feichtinger, and four anonymous referees for helpful comments and advice. 


\section{A Appendix}

\section{A.1 Derivation of equation 4}

As already stated above, the Lorenz curve $L(F(z))$ plots cumulative shares of income as a function of cumulative population shares $F(z)$ when individuals are ranked in increasing order of income $z$ (Lam 1988, p. $143 \mathrm{ff}$.).

Consequently, the Lorenz curve is a monotonically increasing and convex function, where $L(0)=0$ and $L(1)=1$ (see Chotikapanich 1993, Ogwang and Rao 1996). Furthermore, the Lorenz curve fulfills (Chotikapanich 1993)

$$
0<L(F(z))<F(z)<1 \text {. }
$$

A mathematical representation of the Lorenz curve can be derived as follows. Given an income distribution function with density function $f(x)$, the horizontal axis of the Lorenz curve is given by the cumulative distribution function

$$
F(z)=\int_{0}^{z} f(x) d x
$$

and the vertical axis is given by the first moment distribution function

$$
L(F(z))=\frac{1}{y} \int_{0}^{z} x f(x) d x=\frac{1}{y}\left[z F(z)-\int_{0}^{z} F(x) d x\right]
$$

where $y$ denotes the first moment of the density function $f$ (i. e., the mean income/entitlement). The second identity has been derived by applying partial integration (see Atkinson 1970, Lam 1988). Differentiating equation (A.3) with respect to $z$ yields

$$
\frac{d L(F(z))}{d z}=\frac{1}{y} z f(z) .
$$

Furthermore, the following condition holds

$$
\frac{d L(F(z))}{d z}=\frac{d L(F(z))}{d F(z)} \frac{d F(z)}{d z}=\frac{d L(F(z))}{d F(z)} f(z) .
$$

Combining equations (A.4) and (A.5) yields

$$
\frac{d L(F(z))}{d F(z)} f(z)=\frac{1}{y} z f(z),
$$

or equivalently

$$
\frac{d L(F(z))}{d F(z)}=\frac{1}{y} z
$$


The last equality establishes the assertion that the slope of the Lorenz curve is inversely proportional to the mean income $y$.

\section{A.2 Proof of proposition 1}

The computation of the steady states is constrained to the case $\tilde{y}>\alpha y$ since the share of the population falling short of the subsistence requirement $G\left(\tilde{y} ; y_{t}\right)$ equals 1 . For $\tilde{y}>\alpha y$ the fixed points have to be determined numerically.

Taking

$$
P_{I, t+1}=P_{I t}=P_{I} \text { and } P_{S, t+1}=P_{S t}=P_{S} \quad \forall t
$$

into account, equations (13)-(14) simplify to

$$
\begin{aligned}
& \left(b_{I}-d\left(y_{I}\right)\right) P_{I}+P_{S}=0 \\
& \left(b_{S}-d\left(y_{S}\right)\right) P_{S}-P_{S}=0 .
\end{aligned}
$$

Eq. (A.9) yields that either the equilibrium number of food-secure population equals zero or that the natural population growth rate of the food-secure population equals one. For plausible parameter values one can disregard the latter case.

Furthermore, Dworak (2002, chapter 5) demonstrates that there is a unique negative relationship between the numbers of food-insecure population and the resource stock for a given number of food-secure population.

Moreover, the equilibrium number of the food-secure population equalling zero implies that either also the food-insecure population equals zero in the equilibrium or the natural population growth rate of the food-insecure equals zero from equation (A.8). However, the right-hand side of the difference equation system (13)-(14) is not defined for a zero total population size.

The condition of zero natural population growth for the food-insecure population implies that the per capita food entitlement of the food-insecure population equals the following expression

$$
y_{I}\left(P_{I}, 0, R\right)=y_{m}+\frac{1}{\delta} \ln \left(\frac{d_{\max }}{b_{I}-d_{\text {nat }}}-1\right) .
$$

The equation above exhibits a unique solution for $R$ taking also the negative relationship between $R$ and $P_{I}$ into account.

For the existence of the fixed point, the equilibrium values have to fulfill the condition $\tilde{y}>\alpha y\left(\hat{P}_{I}, 0, \hat{R}\right)$. However, since the number of food-secure population equals zero, the per capita food entitlement of the food-insecure population equals that of the total population, i. e., $y_{I}=y$. Hence, the condition above may be transformed to

$$
\frac{\tilde{y}}{\alpha}>y_{I}=y_{m}+\frac{1}{\delta} \ln \left(\frac{d_{\max }}{b_{I}-d_{\text {nat }}}-1\right),
$$


which is equal to

$$
b_{I}-d(\tilde{y} / \alpha)>0 .
$$

\section{A.3 Technical details of the bifurcation analysis}

As evident from Fig. 2 and 5, we have either one or three fixed points. In general, the regions of the differing number of fixed points are separated by fold curves, which collide in a Cusp point. Furthermore, the stability of the fixed point changes and a closed invariant curve emerges when passing the Neimark-Sacker curves, which are abbreviated by 'NS' in Fig. 2 and 5, with a '+' or '--' in the subscript denoting whether the Neimark-Sacker bifurcation is subcritical or supercritical, respectively. ${ }^{15}$ Furthermore, the fold and the Neimark-Sacker curve meet tangentially in a strong resonance bifurcation $1: 1$ (denoted by $R 1: 1$ ). ${ }^{16}$

However, at $\tilde{y}=\alpha y_{I}\left(\hat{P}_{I}, 0, \hat{R}\right)$ which is equivalent to

$$
b_{I}-d\left(\frac{\tilde{y}}{\alpha}\right)=0 \text {, }
$$

the right-hand side of the equation system (13)-(15) is not differentiable. Hence, the bifurcation behaviour cannot be inferred from the multipliers of the fixed point and therefore has to be determined by observing orbits.

For instance, passing from region (a) to (e) by crossing $b_{I}-d(\tilde{y} / \alpha)=0$, the unique fixed point loses its stability and simultaneously an attracting closed invariant curve emerges, which is characteristic for a supercritical Neimark-Sacker bifurcation. Furthermore, passing from (b) to (d), the stable fixed point with zero food-secure population and the unstable fixed point collide and vanish similar to a fold bifurcation. Finally, when passing from (c) to (e), it seems that both bifurcations occur, i. e., the stable and the unstable fixed points collide and vanish and a stable closed invariant curve appears.

Furthermore, in region (e) the fixed point is unstable and the orbits converge to the closed invariant curve. Moving from region (e) to region (d), the fixed point undergoes a sub-critical Neimark-Sacker bifurcation, where the fixed point becomes stable and an unstable closed invariant curve emerges (Kutsnetsov 1998). Shortly after passing the sub-critical Neimark-Sacker bifurcation, however, the track of the closed invariant curve is lost and the unique equilibrium is globally stable. The latter is indi-

15 At a supercritical Neimark-Sacker bifurcation the fixed point loses its stability and a stable closed invariant curve emerges. In contrast, at a subcritical Neimark-Sacker bifurcation, the fixed point becomes stable and an unstable closed invariant emerges.

16 Furthermore, an infinite number of Arnold tongues may be rooted along the Neimark-Sacker curve. According to Kutsnetsov (1998), there may also exist transversal homoclinic structures in an exponentially narrow parameter region bounded by two smooth curves of homoclinic tangencies. In addition, the fold curves delimiting the Arnold tongues accumulate on these homoclinic tangencies curves (Kutsnetsov 1998). 
cated by a dashed curve in Fig. 2 and 5. It seems that there occurs a collision of the unstable and the stable closed invariant curves leaving the then globally stable fixed point. But contrary to continuous-time models, such a bifurcation of closed invariant curves is not well-defined away from the Neimark-Sacker bifurcation, though "there exists a complicated (but narrow) bifurcation set related to the 'collision' of two invariant curves" (Kutsnetsov 2002).

Another limitation on the bifurcation analysis is imposed by the fact that the right-hand side of the equation system (13)-(15) is not differentiable at all points and thus backward iteration is not possible everywhere. Therefore, further unstable closed invariant curves may exist but cannot be detected or, if their existence is detected by subcritical Neimark-Sacker bifurcations, they cannot be continued as the parameters change.

\section{References}

Anand, S. 1983. Inequality and Poverty in Malaysia. London: Oxford University Press.

Anderies, J. M. 1998. Culture and human agro-ecosystem dynamics: The Tsembaga of New Guinea. Journal of Theoretical Biology 192(4), pp. 515-530.

Anderies, J. M. 2003. Economic development, demographics, and renewable resources: a dynamical systems approach. Environment and Development Economics 8(2), pp. 219-246.

Ascher, W. and R. Healy. 1990. Natural Resource Policymaking in Developing Countries: Environment, Economic Growth, and Income Distribution. Durham: Duke University Press.

Atkinson, A. B. 1970. On the measurement of inequality. Journal of Economic Theory 2 , pp. 244-263.

Beaumont, P. M. and R. T. Walker. 1996. Land degradation and property regimes. Ecological Economics 18(1), pp. 55-66.

Brander, J. A. and M. S. Taylor. 1998. The simple economics of Easter Island: A Ricardo-Malthus model of renewable resource use. The American Economic Review 88(1), pp. 119-138.

Chotikapanich, D. 1993. A comparison of alternative functional forms for the Lorenz curve. Economic Letters 41(2), pp. 129-138.

Clark, C. W. 1990. Mathematical Bioeconomics: The Optimal Management of Renewable Resources. New York: J. Wiley.

Cleaver, K. M. and G. A. Schreiber. 1994. Reversing the Spiral: The Population, Agriculture and Environment Nexus in Sub-Saharan Africa. Washington, DC: The World Bank.

Dasgupta, P. 1993. An Inquiry into Well-Being and Destitution. Oxford: Oxford University Press. 
Dasgupta, P. and D. Ray. 1987. Inequality as a determinant of malnutrition and unemployment: policy. The Economic Journal 97(386), pp. 177-188.

Dworak, M. 2002. Population Growth, Food Security and Land Degradation: Modeling the Nexus in Sub-Saharan Africa. PhD Thesis. Vienna University of Technology.

Filmer, D. and L. Pritchett. 1997. Environmental Degradation and the Demand for Children: Searching for the Vicious Circle. Poverty, Environment, and Growth Working Paper No. 2. Washington, DC: The World Bank.

Food and Agriculture Organization. 1999. The state of food insecurity in the World 1999 [online]. Rom: Food and Agriculture Organization. Available from: http://www.fao.org.

Food and Agriculture Organization. 2000. The state of food insecurity in the World 2000 [online]. Rom: Food and Agriculture Organization. Available from: http://www.fao.org.

Food and Agriculture Organization. 2001. The state of food insecurity in the World 2001 [online]. Rom: Food and Agriculture Organization. Available from: http://www.fao.org.

Galor, O. and J. Zeira. 1993. Income distribution and macroeconomics. Review of Economic Studies 60(1), pp. 35-52.

Jolly, C. L. 1994. Four theories of population change and the environment. Population and Environment 16(1), pp. 61-90.

Kremer, M. and D. Chen. 1999. Income-distribution dynamics with endogenous fertility. American Economic Review 89(2), pp. 155-160.

Kutsnetsov, Y. A. 1998. Elements of Applied Bifurcation Theory. 2 ed. New York: Springer.

Kutsnetsov, Y. A. 2002. Personal Communication.

Lam, D. 1988. Lorenz curves, inequality, and social welfare under changing population composition. Journal of Policy Modeling 10(1), pp. 141-162.

Loughran, D. and L. Pritchett. 1997. Environmental Scarcity, Resource Collection, and the Demand for Children in Nepal. Poverty, Environment, and Growth Working Paper No. 19. Washington, DC: The World Bank.

Lutz, W. and S. Scherbov. 2000. Quantifying vicious circle dynamics: The PEDA model for population, environment, development and agriculture in African countries. In: Dockner, E. J., R. F. Hartl, M. Luptačik, and G. Sorger (eds). Optimization, Dynamcis, and Economic Analysis: Essays in Honor of Gustav Feichtinger. Heidelberg: Physica-Verlag, pp. 311-322.

Lutz, W., S. Scherbov, A. Prskawetz, M. Dworak, and G. Feichtinger. 2002. Population, natural resources and food security: Lessons from comparing full and reduced form models. In: Lutz, W., A. Prskawetz, and W. C. Sanderson (eds). Population and Environment: Methods of Analysis. Supplement to Population and Development Review 28. New York: Population Council, pp. 199-224. 
Lutz, W., S. Scherbov, P. K. Makinwa-Adebusoye, and G. Reniers. 2004. Population-environment-development-agriculture interactions in Africa: a case study on Ethiopia. In: Lutz, W. and W. Sanderson (eds). The End of World Population Growth, Human Capital and Sustainable Development in the $21^{\text {st }}$ Century. London: Earthscan.

Nerlove, M. and A. Meyer. 1997. Endogenous fertility and the environment: A parable of firewood. In: Dasgupta, P. and K.-G. Mäler (eds). The Environment and Emerging Development Issues 2. Oxford: Clarendon Press, pp. 259-282.

Ogwang, T. and U. L. Gouranga Rao. 1996. A new functional form for approximating the Lorenz curve. Economics Letters 52(1), pp. 21-29.

Prskawetz, A., G. Steinmann and G. Feichtinger. 2000. Human capital, technological progress and the demographic transition. Mathematical Population Studies 7(4), pp. 343-363.

Prskawetz, A., M. Winkler-Dworak, and G. Feichtinger. 2003. Production, distribution and insecurity of food: A dynamic framework. Structural Change and Economic Dynamics 14(3), pp. 317-337.

Rothschild, M. and J. E. Stiglitz. 1970. Increasing risk: I. a definition. Journal of Economic Theory 2, pp. 188-204.

Rothschild, M. and J. E. Stiglitz. 1973. Some further results on the measurement of inequality. Journal of Economic Theory 6(2), pp. 188-204.

Ruthenberg, H. 1980. Farming Systems in the Tropics. Oxford: Clarendon Press.

Strauss, J. 1986. Does better nutrition raise farm productivity. Journal of Political Economy 94(2), pp. 297-320.

Strulik, H. 1995. Die Interdependenz von Bevölkerungs- und Wirtschaftsentwicklung in neoklassischen Wachstumsmodellen. PhD Thesis. Universität Göttingen.

Young, A. 1998. Land Resoures: Now and for the future. Cambridge: Cambridge University Press. 